\title{
Transport of intensity and spectrum for partially coherent fields
}

\author{
Adam M. Zysk, ${ }^{1}$ Robert W. Schoonover, ${ }^{2,4}$ P. Scott Carney, ${ }^{2}$ and Mark A. Anastasio ${ }^{3, *}$ \\ ${ }^{1}$ Department of Electrical and Computer Engineering, Medical Imaging Research Center, \\ Illinois Institute of Technology, Chicago, Illinois 60616, USA \\ ${ }^{2}$ Beckman Institute for Advanced Science and Technology, Department of Electrical and Computer Engineering, \\ University of Illinois at Urbana-Champaign, Urbana, Illinois 61801, USA \\ ${ }^{3}$ Department of Biomedical Engineering, Medical Imaging Research Center, Illinois Institute of Technology, \\ Chicago, Illinois 60616, USA \\ ${ }^{4}$ Currently at the Illinois Institute of Technology \\ *Corresponding author: anastasio@iit.edu
}

Received March 16, 2010; revised May 27, 2010; accepted May 27, 2010; posted June 9, 2010 (Doc. ID 125559); published June 25, 2010

\begin{abstract}
Transport-of-intensity and transport-of-spectrum equations are derived using the coherent mode decomposition for paraxial fields having an arbitrary state of coherence. We give a simple example that demonstrates the difference between a partially coherent and a fully coherent transport of intensity or spectrum. The results presented here may be used to estimate the intensity response in a variety of phase-contrast imaging modalities and may form the basis for improved phase-retrieval techniques. (c) 2010 Optical Society of America

OCIS codes: $\quad 030.0030,030.4070,110.4980,350.5030$.
\end{abstract}

Phase contrast is commonly exploited to circumvent the low absorption contrast of semitransparent samples in a wide range of imaging techniques, including optical microscopy [四], x-ray imaging [[2], and transmission electron microscopy [3]. In quantitative phase-contrast imaging techniques, phase-retrieval methods are employed to compute an image that depicts the projected refractive index distribution of a sample. Assuming perfectly coherent illumination, phase-retrieval methods have been developed based on the transport-of-intensity equation (TIE) and other physical models that relate the field phase to intensity measurements [四]. In practice, of course, only partially coherent fields are available, and performing phase retrieval based on the assumption of a perfectly coherent illuminating field can yield erroneous results.

In this Letter, we make use of the coherent mode decomposition [5-8] to derive a generalization of the TIE that is valid for spatially partially coherent paraxial fields in which the modal phases are governed by the eikonal equation [7]. In previous works [9,[10], a model has been presented that is not derived from the Wolf equations but, instead, introduces a heuristic "generalized phase" that reduces to the solution to the eikonal equation in the limit that the field is fully spatially coherent. While this technique may yield reasonable results in some situations, such as in synchrotron radiation when the overwhelming majority of power is concentrated in one mode [11]], multiple modes exist in partially coherent fields, e.g., those from x-ray tube sources. The phase and amplitude from each mode contributes to the field intensity and, therefore, the TIE must incorporate them all.

Consider first a deterministic monochromatic field that satisfies the paraxial wave equation, i.e., $U(\mathbf{r}, \omega)=$ $V(\mathbf{r}, \omega) \exp (i k z)$, where

$$
\left[2 i k \frac{\partial}{\partial z}+\nabla_{\perp}^{2}\right] V(\mathbf{r}, \omega)=0
$$

and $\nabla_{\perp}^{2}$ denotes the transverse Laplacian. By writing Eq. (11) as $\hat{L} V=0$, the construction of the quantity $V^{*} \hat{L} V-V[\hat{L} V]^{*}$ results in the local relation

$$
\frac{\partial S(\mathbf{r}, \omega)}{\partial z}=-\frac{1}{k} \nabla_{\perp} \cdot S(\mathbf{r}, \omega) \nabla_{\perp} \psi(\mathbf{r}, \omega),
$$

where $S=U^{*} U=V^{*} V$ is the Fourier spectrum of the field and $\psi=\arg (V)$ is the phase of the field. This equation relates the local rate of change of the spectrum in the approximate direction of wave propagation to the transverse rates of change of the spectrum and the phase of

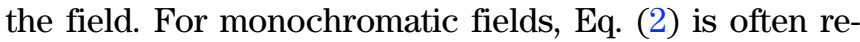
ferred to as the TIE. It should be noted that Eq. (2) is actually a transport-of-spectrum equation and that, generally, no simple relationship exists between the spectrum and intensity for polychromatic fields.

Although many treatments of energy transport for imaging applications are developed by using deterministic fields like the one above, realizable fields and sources are stochastic (random) processes. Random fluctuations in the field induce partial coherence between values of the field at pairs of points and times (or frequencies) [6]. In general, such fields do not have well-defined Fourier transforms and, thus, the Fourier spectrum in Eq. (2) does not exist, except in the generalized sense. For stationary ergodic fields, however, the Fourier spectrum may be replaced by a power spectral density.

The fields in Eq. (11) are not directly accessible, being realizations of a random process. In the spectral domain, the measurable quantity of interest is the cross-spectral density (CSD):

$$
W\left(\mathbf{r}_{1}, \mathbf{r}_{2}, \omega\right)=\left\langle U^{*}\left(\mathbf{r}_{1}, \omega\right) U\left(\mathbf{r}_{2}, \omega\right)\right\rangle_{\omega},
$$

where $\langle\cdot\rangle_{\omega}$ denotes the ensemble average of frequencydomain realizations. The power spectral density, $S(\mathbf{r}, \omega)=W(\mathbf{r}, \mathbf{r}, \omega)$, gives the power per frequency in the optical field. The CSD may be expressed in a coherent mode decomposition [5, [2]]: 


$$
W\left(\mathbf{r}_{1}, \mathbf{r}_{2}, \omega\right)=\sum_{m=1}^{M} \gamma_{m}(\omega) \phi_{m}^{*}\left(\mathbf{r}_{1}, \omega\right) \phi_{m}\left(\mathbf{r}_{2}, \omega\right),
$$

where $\gamma_{m}$ are spectral weights, $\phi_{m}$ are the so-called coherent modes that are statistically independent and satisfy the Helmholtz equation (or an appropriate approximation), and $M$ is the total number of coherent modes. It should be noted that the use of the power spectral density and CMD is based on an assumption of stationary ergodic fields. As in the deterministic case, the $\phi_{m}$ can be written as

$$
\phi_{m}(\mathbf{r}, \omega)=a_{m}(\mathbf{r}, \omega) e^{i \psi_{m}(\mathbf{r}, \omega)},
$$

where $\psi_{m}$ is the real-valued phase of mode $m$. While the deterministic coherent modes have a well-defined phase, it is important to stress that the random field does not. The only measurable phase related to the partially coherent field is that of the CSD.

From Eq. (四), we find the spectrum of the field:

$$
S(\mathbf{r}, \omega)=\sum_{m=1}^{M} \gamma_{m}(\omega) D_{m}(\mathbf{r}, \omega),
$$

where $D_{m}(\mathbf{r}, \omega)=a_{m}^{2}(\mathbf{r}, \omega)$. Substitution into Eq. (27) gives the transport-of-spectrum equation for partially coherent fields:

$$
\frac{\partial S(\mathbf{r}, \omega)}{\partial z}=-\frac{1}{k} \sum_{m=1}^{M} \gamma_{m}(\omega) \nabla_{\perp} \cdot\left[D_{m}(\mathbf{r}, \omega) \nabla_{\perp} \psi_{m}(\mathbf{r}, \omega)\right]
$$

The TIE for partially coherent fields is found by integrating Eq. (ד) over $\omega$ :

$$
\frac{\partial I(\mathbf{r})}{\partial z}=-\sum_{m=1}^{M} \int \mathrm{d} \omega \frac{1}{k} \gamma_{m}(\omega) \nabla_{\perp} \cdot\left[D_{m}(\mathbf{r}, \omega) \nabla_{\perp} \psi_{m}(\mathbf{r}, \omega)\right] .
$$

Equations (7D) and (8) are the main results of this work. For a random paraxial field in an arbitrary state of partial coherence, they relate variations along the optical axis in the spectrum or intensity to the coherent modes of the random field. No further simplifications in these equations may be made without additional assumptions regarding the physical or statistical characteristics of the field.

Equation (8) may be reduced to the results of other intensity propagation models for polychromatic fields [10, [3, [14], for the case that the field is geometric. In the geometrical limit, the modes take the form given in Eq. (5) but with $a_{m}$ and $\psi_{m}$ independent of frequency. We write $D_{m}(\mathbf{r}, \omega) \rightarrow \bar{D}_{m}(\mathbf{r})$ and $\psi_{m}(\mathbf{r}, \omega) \rightarrow k \bar{\psi}_{m}(\mathbf{r})$. Equation (17) reduces to

$$
\frac{\partial S(\mathbf{r}, \omega)}{\partial z}=-\nabla_{\perp} \cdot \sum_{m=1}^{M} \gamma_{m}(\omega) \bar{D}_{m}(\mathbf{r}) \nabla_{\perp} \bar{\psi}_{m}(\mathbf{r}),
$$

and Eq. (8) reduces to

$$
\frac{\partial I(\mathbf{r})}{\partial z}=-\nabla_{\perp} \cdot \sum_{m=1}^{M} P_{m} \bar{D}_{m}(\mathbf{r}) \nabla_{\perp} \bar{\psi}_{m}(\mathbf{r})
$$

where

$$
P_{m}=\int \mathrm{d} \omega \gamma_{m}(\omega)
$$

is the total intensity in each mode.

Note that, when only one mode is relevant, the field can also be regarded as being fully spatially coherent, i.e., $P_{m}=0 \forall m \neq 1$. In this case, Eq. (10) reduces to

$$
\frac{\partial I(\mathbf{r})}{\partial z}=-P_{1} \nabla_{\perp} \cdot \bar{D}_{1}(\mathbf{r}) \nabla_{\perp} \bar{\psi}_{1}(\mathbf{r})
$$

Note that $P_{1} \bar{D}_{1}(\mathbf{r})=I(\mathbf{r})$. This is the result found by Gureyev et al. under the potential scattering model ([10], see, for example, Eq. 1). Additionally, in the case that the field is single mode and $\nabla_{\perp} S \cdot \nabla_{\perp} \bar{\psi}_{1} \ll S \nabla_{\perp}^{2} \bar{\psi}_{1}$, the phase satisfies the Poisson equation [13]:

$$
\nabla_{\perp}^{2} \bar{\psi}_{1}(\mathbf{r}, \omega)=-S^{-1}(\mathbf{r}, \omega) \frac{\partial S(\mathbf{r}, \omega)}{\partial z} .
$$

Equations (12) and (13) can be applied to fields that are well approximated by a single coherent mode. The TIE has many important applications in fields such as optical microscopy, for example, where it serves as the basis for phase-retrieval algorithms [13,[15]. As an example of a single-mode field to which Eqs. (12) and (13) apply, consider a statistically stationary point source. For a single point source $S=1 / r^{2}$, where $r$ is the distance from the source to the observation point, and the right-hand side of Eq. (13) evaluates to $-S^{-1} \partial S / \partial z=2 z / r^{2}$. The phase of the field radiating from the point source is given by $\bar{\psi}_{1}=r$, and, so, the left-hand side of Eq. (13) evaluates to $\nabla_{\perp}^{2} \bar{\psi}_{1}=\left(r^{2}+z^{2}\right) / r^{3}$. Within the paraxial zone, $r \approx z$ and Eq. (13) is satisfied by these two expressions.

Consider a simple example in which Eq. (D) is valid but Eqs. (27) and (12) do not apply, such as the field generated by two incoherent point sources. The resulting CSD has the coherent mode decomposition

$$
W\left(\mathbf{r}^{\prime}, \mathbf{r}^{\prime \prime}, \omega\right)=\sum_{m=1}^{2} \gamma_{m}(\omega) \frac{e^{-i k\left|\mathbf{r}^{\prime}-\mathbf{r}_{m}\right|}}{\left|\mathbf{r}^{\prime}-\mathbf{r}_{m}\right|} \frac{e^{i k\left|\mathbf{r}^{\prime \prime}-\mathbf{r}_{m}\right|}}{\left|\mathbf{r}^{\prime \prime}-\mathbf{r}_{m}\right|},
$$

where $\mathbf{r}_{m}$ is the location of the $m$ th radiator and $\gamma_{m}(\omega)$ denotes the spectrum of each point radiator. The righthand side of Eq. (13) can be estimated from measurements of the spectral density in closely adjacent planes normal to the $z$ axis, and, in this example, can be directly computed as

$$
-S(\mathbf{r}, \omega)^{-1} \frac{\partial S(\mathbf{r}, \omega)}{\partial z}=\frac{\frac{2 \gamma_{1}(\omega) z}{\left|\mathbf{r}-\mathbf{r}_{1}\right|^{4}}+\frac{2 \gamma_{2}(\omega) z}{\left|\mathbf{r}-\mathbf{r}_{2}\right|^{4}}}{\frac{\gamma_{1}(\omega)}{\left|\mathbf{r}-\mathbf{r}_{1}\right|^{2}}+\frac{\gamma_{2}(\omega)}{\left|\mathbf{r}-\mathbf{r}_{2}\right|^{2}}} .
$$

In Fig. 1, the results of inverting Eq. (13) for three cases are plotted as a function of the $x$ coordinate in the detection plane. In the first case, Eq. (13) is evaluated for the 


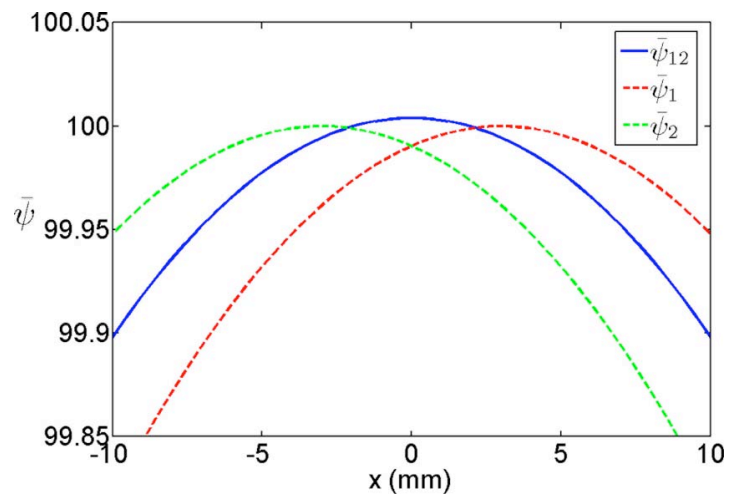

Fig. 1. (Color online) Plot of the retrieved phases when the wave field is assumed to be fully coherent, found by inverting Eq. (13), on the line $z=100 \mathrm{~mm}$ and $y=0$ for three cases: $\gamma_{1}(\omega)=1$ and $\gamma_{2}(\omega)=0\left(\bar{\psi}_{1}\right), \gamma_{1}(\omega)=0$ and $\gamma_{2}(\omega)=1\left(\bar{\psi}_{2}\right)$, and $\gamma_{1}(\omega)=1$ and $\gamma_{2}(\omega)=0\left(\bar{\psi}_{12}\right)$.

field described by Eq. (14) when $\gamma_{1}(\omega)=1$ and $\gamma_{2}(\omega)=0$ (denoted $\left.\bar{\psi}_{1}\right)$, in the second case $\gamma_{1}(\omega)=0$ and $\gamma_{2}(\omega)=1$ $\left(\right.$ denoted $\left.\bar{\psi}_{2}\right)$, and in the third case $\gamma_{1}(\omega)=1$ and $\gamma_{2}(\omega)=$ 1 (denoted $\bar{\psi}_{12}$ ). It can be seen that, when the field is multimode (the field associated with $\psi_{12}$ ), the so-called retrieved phase does not match the accumulated phase of either mode (nor is it the average or sum of the phases of the two modes). Moreover, $\bar{\psi}_{12}$ is not associated with any physical optical path length travelled. As a result, phase retrieval based on Eq. (12), i.e., the implicit assumption of spatial coherence, returns nonphysical results.

To accurately find the phase of each coherent mode, phase-retrieval methods based on Eq. (77) or Eq. (8) must be developed and employed. It should be emphasized that there is no single phase to be recovered from a partially coherent field. Instead, one may hope to recover the amplitude and phase of each coherent mode. In contrast to previously proposed methods, which assumed the existence of a single coherent mode and a single phase, this process inherently requires at least $M$ measurements when $M$ coherent modes exist.

One possible phase recovery strategy involves making measurements at multiple frequencies and solving a system of linear equations based on Eq. (D). Since spectrally and spatially resolved measurements can be experimentally challenging in the x-ray regime, we propose an alternative scheme. By modifying the coherence properties of the illuminating field, it may be possible to construct a system of equations using Eq. (8) that can be solved to arrive at the phase of each mode. Under the assumptions inherent in fully coherent TIE [see Eq. (2)], [四 conventional phase-retrieval methods may be applied to the solution of the system of linear equations [13].
In summary, we have introduced new TIE and transport-of-spectrum equations that relate variations in intensity or spectrum to the coherent modes of the CSD. These results are generally applicable to intensity transport in spatially partially coherent fields, i.e., fields described by more than one coherent mode. Such fields are produced by multimode lasers, extended thermal sources, and arrays of single-mode sources, and are, generically, the fields encountered in practice. As in recent studies of coherent diffractive imaging [ [16], these models may provide a framework for the development of phase-retrieval methods for use with partially coherent paraxial fields.

R. W. S. and P. S. C. acknowledge support from the Grainger Foundation for Emerging Technologies. A. M. Z. acknowledges support from the National Institutes of Health (NIH) (NCI, 1 F32 CA136102). M. A. A. acknowledges support from the National Science Foundation (NSF) (CBET-0546113 and CBET-0854430) the NIH (NIBIB, 1 R01 EB009715).

\section{References}

1. A. H. Bennett, H. Osterberg, H. Jupnik, and O. W. Richards, Phase Microscopy: Principles and Applications (Wiley, 1951).

2. S. C. Mayo, T. J. Davis, T. E. Gureyev, P. R. Miller, D. Paganin, A. Pogany, A. W. Stevenson, and S. W. Wilkins, Opt. Express 11, 2289 (2003).

3. T. C. Petersen, V. J. Keast, and D. M. Paganin, Ultramicroscopy 108, 805 (2008).

4. M. R. Teague, J. Opt. Soc. Am. 73, 1434 (1983).

5. E. Wolf, J. Opt. Soc. Am. 72, 343 (1982).

6. E. Wolf, Introduction to the Theory of Coherence and Polarization of Light (Cambridge U. Press, 2007).

7. A. M. Zysk, P. S. Carney, and J. C. Schotland, Phys. Rev. Lett. 95, 043904 (2005).

8. R. W. Schoonover, A. M. Zysk, P. S. Carney, J. C. Schotland, and E. Wolf, Phys. Rev. A 77, 043831 (2008).

9. K. A. Nugent, T. E. Gureyev, D. F. Cookson, D. Paganin, and Z. Barnea, Phys. Rev. Lett. 77, 2961 (1996).

10. T. E. Gureyev, D. M. Paganin, A. W. Stevenson, S. C. Mayo, and S. W. Wilkins, Phys. Rev. Lett. 93, 068103 (2004).

11. S. Flewett, H. M. Quiney, C. Q. Tran, and K. A. Nugent, Opt. Lett. 34, 2198 (2009).

12. B. J. Davis and R. W. Schoonover, Opt. Lett. 34, 923 (2009).

13. T. E. Gureyev, A. Pogany, D. M. Paganin, and S. W. Wilkins, Opt. Commun. 231, 53 (2004).

14. D. Paganin and K. A. Nugent, Phys. Rev. Lett. 80, 2586 (1998).

15. A. Barty, K. A. Nugent, D. Paganin, and A. Roberts, Opt. Lett. 23, 817 (1998).

16. L. W. Whitehead, G. J. Williams, H. M. Quiney, D. J. Vine, R. A. Dilanian, S. Flewett, K. A. Nugent, A. G. Peele, E. Balaur, and I. McNulty, Phys. Rev. Lett. 103, 243902 (2009). 